\title{
Effective enhancement of X-ray-induced apoptosis in human cancer cells with mutated $p 53$ by siRNA targeting XIAP
}

\author{
KEN OHNISHI $^{1}$, YOKO NAGATA ${ }^{2}$, AKIHISA TAKAHASHI ${ }^{1}$, \\ SHIGEKI TANIGUCHI ${ }^{2}$ and TAKEO OHNISHI ${ }^{1}$
}

\author{
Departments of ${ }^{1}$ Biology and ${ }^{2}$ Thoracic and Cardiovascular Surgery, Nara Medical \\ University School of Medicine, 840 Shijo-cho, Kashihara, Nara 634-8521, Japan
}

Received December 28, 2007; Accepted February 4, 2008

\begin{abstract}
The X-chromosome-linked inhibitor of the apoptosis protein (XIAP) is known to be an inhibitory factor for caspase-3. The aim of our study was to see whether radiation-induced apoptosis is enhanced by RNA interference targeting the XIAP through an elevation of caspase- 3 activity, and whether the effect of XIAP depression depends on the p53 status of cancer cells. Two types of transformed human cultured non-small cell lung cancer cells (H1299) were used: wild-type p53-transfected cells (H1299/wtp53) and mutated p53-transfected cells (H1299/mp53). When 21mer siRNA targeting XIAP (XIAP-siRNA) was transfected into these cells using liposomes, a suppression of the constitutive XIAP protein expression was observed. XIAPsiRNA enhanced radiation sensitivity in H1299/wtp53 and in $\mathrm{H} 1299 / \mathrm{mp} 53$ cells and was very effective in H1299/mp53 cells. Radiation-induced apoptosis and the activation of caspase-3 were more elevated by XIAP-siRNA in the H1299/mp53 cells than in H1299/ wt $p 53$. These results suggest that $X I A P$-siRNA is a possible candidate for a radiation sensitizer in cancer radiotherapy, especially in cells with mutated $p 53$.
\end{abstract}

\section{Introduction}

It is important to search for predictive indicators which can help clinicians improve the outcome and efficacy of cancer therapy. In particular, signaling factors involved in apoptosis induction are now being recognized as possible tools in cancer therapy, since apoptosis is a beneficial physiological response which can result in cell death without inflammation. The efficacy of radiation, heat, or anti-cancer agents on cancer cells can be affected by p53 status through the induction of apoptosis

Correspondence to: Dr Takeo Ohnishi, Department of Biology, Nara Medical University School of Medicine, Kashihara, Nara 634-8521, Japan

E-mail: tohnishi@naramed-u.ac.jp

Key words: radiation, apoptosis, siRNA, X-chromosome-linked inhibitor of the apoptosis protein, $p 53, \mathrm{X}$-ray
(1-4). Several studies using cancer cell lines (other than the ones used here) transfected with mp53 have reported that the constitutive expression of mp53 proteins results in a resistance to radiation (5-9). Furthermore, it has been clinically demonstrated that cervical cancer patients with wtp53 have high survival rates after radiotherapy when compared with cervical cancer patients with mp53 (10). Bax and Bcl-2, which are involved in $p 53$-dependent signal transduction pathways, are good clinical candidates for predictive indicators (11). It appears likely that cancer radiation therapy based on $p 53$ gene status might improve the outcome of cancer therapies in patients with normal p53 function. On the other hand, it is necessary to develop therapeutic strategies which can be used with mp53 cancer cells with more advanced cancer therapies. A strategy of radiation enhancement regardless of cellular p53 status is currently the focus of intense research. The aim of this study was to examine whether it was possible to enhance radiation-induced apoptosis by depressing a survival signaling factor, XIAP and how the effect of XIAP depression might depend on the p53 status in the human lung cancer cells differing only in their $p 53$ status.

The activation of cell survival signaling pathways is considered to contribute to cancer therapy resistance (12-14). According to this model, defects in cell survival signaling pathways correlate with effective radiation cancer therapy. XIAP is involved in cell survival and plays a pivotal role in cancer progression (15). High expression levels of XIAP are clinically associated with poor survival prognosis in patients (16). Based on these facts, the down-regulation of XIAP expression could be advantageous in cancer therapy. A recent report shows that the down-regulation of XIAP expression by $X I A P$-siRNA resulted in the sensitization to radiation (1). Other reports also indicate that the down-regulation of XIAP expression by XIAP-siRNA (17) or antisense oligonucleotides (18) led to cancer cell sensitization to radiation. XIAP is known to inhibit the induction of apoptosis by blocking caspase-3 activation $(19,20)$. However, the relationship between the regulation of XIAP expression and the frequency of radiation-induced apoptosis has not been fully examined in cancer radiation therapy. The down-regulation of XIAP expression should contribute to improved radiation cancer therapy. Notably, the present study demonstrated that $X I A P$-siRNA enhanced radiation sensitivity more effectively in $\mathrm{m} p 53$ cancer cells than in wt $p 53$ cancer cells. 


\section{Materials and methods}

Cell culture. Human lung cancer H1299 cells transfected with pC53-SN3 containing the wtp53 gene, or pC53-248 containing the mp53 gene (Arg to Trp at codon 248) were used in this study (1). The transcription of wtp53 and mp53 genes was confirmed in stable clones of these cells using RT-PCR RFLP and the translation of the p53 protein was confirmed using Western blot analysis (21). Cell culture conditions have been described previously (1). Cells were irradiated in DMEM-10 with a $150 \mathrm{kVp}$ X-ray generator (Model MBR-1520R, Hitachi, Tokyo, Japan) and cultured for the indicated postirradiation periods at $37^{\circ} \mathrm{C}$.

Preparation and transfection of siRNA. Double strand 21-mer siRNA targeted for XIAP (XIAP-siRNA) and a negative control siRNA were provided commercially (B-Bridge International, Inc., Sunnyvale, CA). The sequence of XIAPsiRNA was GTAAAATGCAAGTGGCAAATT. The sequence of the XIAP-siRNA negative control siRNA was TATTCGC GCGTATAGCGGTTT. XIAP-siRNA (200 nM final concentration) was transfected into the H1299/wtp53 and H1299/ mp53 cells with liposomes and an enhancer (Targeting Systems, Santee, CA) according to the manufacturer's protocol. Cells treated with XIAP-siRNA were incubated for $48 \mathrm{~h}$ at $37^{\circ} \mathrm{C}$ and then irradiated or plated for colony formation assays.

Transfection efficiency of XIAP-siRNA. To measure the transfection efficiency of the XIAP-siRNA, the XIAP-siRNA was labeled using a Silencer siRNA labeling kit (Ambion) according to the manufacturer's protocol as described elsewhere (1). The labeled XIAP-siRNA was transfected using liposomes. For a quantitative estimation of $\mathrm{Cy} 3$ positive cells, 40-70 cells were evaluated in three independent random microscopic fields and a total of 120-210 cells were evaluated for each concentration of XIAP-siRNA.

Colony formation assay. The fraction of cells surviving irradiation was determined using a clonogenic assay as described elsewhere (1). In brief, after the transfection of $X I A P$-siRNA, a total of $5 \times 10^{2}$ or $1 \times 10^{3}$ cells were plated in two T25 flasks per experiment. The cells were irradiated $6 \mathrm{~h}$ after plating and after 14 days of incubation, colonies were fixed with $100 \%$ methanol for several minutes and stained with a $2 \%$ Giemsa solution (Merck \& Co., Inc., Rahway, NJ). The plating efficiency was $\sim 75 \%$ in the H1299/wtp53 and H1299/mp53 cells. Three independent experiments were performed for each measurement.

Western blot analysis. Western blot analysis was described in detail previously (1). Transfer membranes were incubated with an appropriate antibody: an anti-XIAP polyclonal antibody (Cell Signaling Technology Inc.), an anti-active caspase-3 polyclonal antibody (Abcam, Cambridge, UK), an anti-cleaved PARP polyclonal antibody (Promega, Madison, WI) and an anti-actin polyclonal antibody (Santa Cruz Biotechnology Inc.). For visualization of the bands, a horseradish peroxidaseconjugated anti-rabbit IgG antibody (Amersham Pharmacia Biotech Inc., Piscataway, NJ) was used for anti-XIAP, antiactive caspase- 3 and anti-cleaved PARP antibodies and a horseradish peroxidase-conjugated anti-goat IgG antibody (Zymed Laboratories Inc.) was used for anti-actin. Actin was used as a control to ensure that the same amount of protein was loaded in each lane. Western blots were performed at least two times to verify the results.

Immunofluorescent staining. Cells $\left(\sim 10^{4}\right.$ cells $)$ were plated on glass slides and $\mathrm{X}$-irradiated in the same manner as described previously. After irradiation and incubation for $24 \mathrm{~h}$ at $37^{\circ} \mathrm{C}$, cells were rinsed twice with PBS, fixed with cold $100 \%$ methanol for $1 \mathrm{~min}$, washed with PBS three times and then permeabilized for 2 min with a solution containing $0.1 \%$ Triton X-100. Then, the cells were washed with PBS and incubated with an anti-caspase-3 polyclonal antibody (Abcam) for $1 \mathrm{~h}$ at $37^{\circ} \mathrm{C}$. After this incubation, cells were washed with PBST three times and then incubated with Alexa 488 (Nacalai Tesque, Kyoto, Japan) for $1 \mathrm{~h}$ at $37^{\circ} \mathrm{C}$. After incubation, the cells were washed with PBST and covered with a glass cover slip.

Analysis of apoptosis. For detection of apoptotic bodies, cells were fixed with $1 \%$ glutaraldehyde (Nacalai Tesque) in PBS at $4^{\circ} \mathrm{C}$, washed with PBS, stained with $0.2 \mathrm{mM}$ Hoechst 33342 (Sigma Chemical Co.) and then observed under a fluorescence microscope.

\section{Results}

Transfection efficiency of XIAP-siRNA. Transfection efficiency of XIAP-siRNA was estimated with the use of Cy3-labeled $X I A P$-siRNA in the H1299/wtp53 and H1299/mp53 cells. The transfection efficiency of siRNA was $80-85 \%$ at $48 \mathrm{~h}$ after transfection with $200 \mathrm{nM}$ of Cy3-labeled XIAP-siRNA (data not shown). No increased transfection efficiency was observed if concentrations $>200 \mathrm{nM}$ of siRNA were used. After these preliminary experiments, each cell was transfected with $200 \mathrm{nM} X I A P$-siRNA to achieve the maximal transfection efficiency.

Sensitivity of $H 1299$ cells to X-rays. The clonogenic survival of cells was measured to determine the X-ray sensitivity of the H1299/wtp53 and H1299/mp53 cells transfected with negative control siRNA or XIAP-siRNA (200 nM) (Fig. 1a). When negative control siRNA-transfected cells were irradiated with X-rays (12 Gy), a difference in sensitivity to X-rays was observed between the H1299/wtp53 ( 2\% cell survival rate) and H1299/mp53 cells ( 6\% survival rate) (t-test, $\mathrm{p}<0.1)$. When the H1299/wtp53 cells were transfected with $X I A P$-siRNA and irradiated, cellular sensitivity to X-rays increased slightly (the survival rate decreased to $\sim 0.9 \%$ ) ( $\mathrm{p}<0.2$ ). When the H1299/mp53 cells were transfected with $X I A P$-siRNA and irradiated, cellular sensitivity to X-rays showed a more pronounced increase (with a survival rate of $\sim 0.02 \%)(\mathrm{p}<0.01)$. These results showed that transfection with $X I A P$-siRNA enhanced the $\mathrm{X}$-ray sensitivity in the cells, though the effect was most pronounced in the H1299/mp53 cells.

Suppression of XIAP expression in XIAP-siRNA-transfected cells. When the H1299/wtp53 cells were transfected with 
a

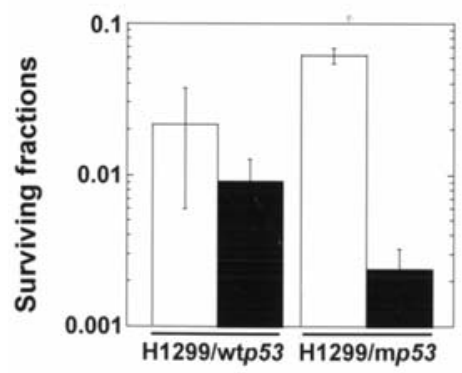

b

H1299/wtp53 $\mathrm{H1299/ \textrm {mp5 }}$

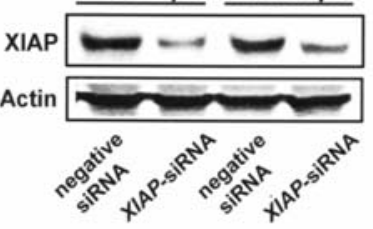

Figure 1. Surviving fractions of H1299 cells transfected with negative control siRNA or XIAP-siRNA after X-irradiation (12 Gy) (a). Effects of $X I A P$-siRNA transfection on XIAP expression (b). Open columns, cells transfected with negative siRNA; solid columns, cells transfected with $X I A P$-siRNA. The constitutive expression of XIAP and actin were analyzed at $48 \mathrm{~h}$ after transfection of negative control siRNA or XIAP-siRNA (200 nM) in whole cell extracts.

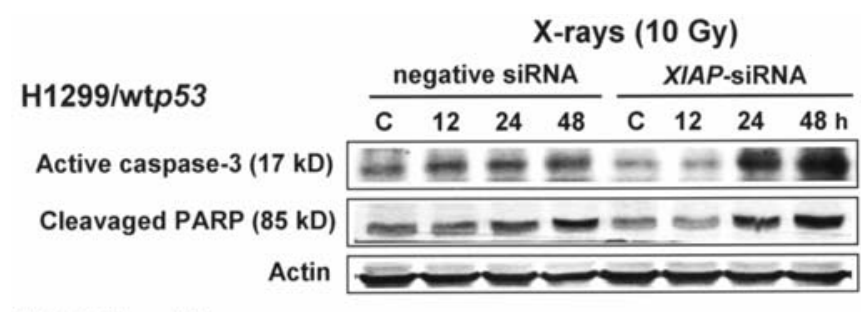

\section{H1299/mp53}

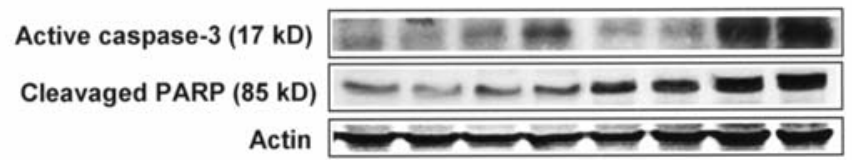

Figure 2. Effects of transfection of XIAP-siRNA on X-ray-induced activation of caspase- 3 and cleavage of PARP. Levels of active caspase- 3 and cleaved PARP were measured in whole cell extracts from H1299/wtp53 and $\mathrm{H} 1299 / \mathrm{mp} 53$ cells at the indicated times after X-irradiation (10 Gy). Cells were transfected with negative control siRNA or XIAP-siRNA $(200 \mathrm{nM})$. The cells were collected at 12, 24 and $48 \mathrm{~h}$ after X-irradiation. Lane C, nonirradiated cells.

$X I A P$-siRNA, constitutive levels of the XIAP protein clearly decreased (Fig. 1b) when compared with negative control siRNA-transfected cells. Similarly, the H1299/mp53 cells transfected with XIAP-siRNA showed decreased constitutive levels of the XIAP protein when compared to negative control siRNA-transfected cells (Fig. 1b).

Enhancement of X-ray-induced activation of caspase-3 and cleavage of PARP in XIAP-siRNA-transfected cells. Activated caspase-3 levels in negative control siRNA-transfected H1299/wtp53 and H1299/mp53 cells increased with time after X-irradiation, when compared with non-irradiated cells (Fig. 2). Consistent with the activation of caspase-3, cleaved PARP increased after irradiation in negative control siRNAtransfected H1299/wtp53 and H1299/mp53 cells (Fig. 2). When the cells were transfected with $X I A P$-siRNA, an enhanced increase of activated caspase- 3 was observed in the H1299/wtp53 and H1299/mp53 cells with time after Xirradiation when compared to non-irradiated cells (Fig. 2). After irradiation, cleaved PARP levels were also enhanced by XIAP-siRNA in the H1299/wtp53 and H1299/mp53 cells negative siRNA XIAP-siRNA

Control
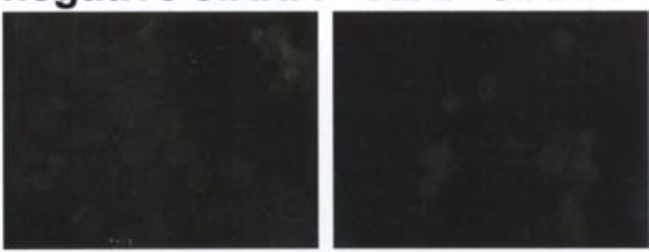

X-ray (10 Gy)
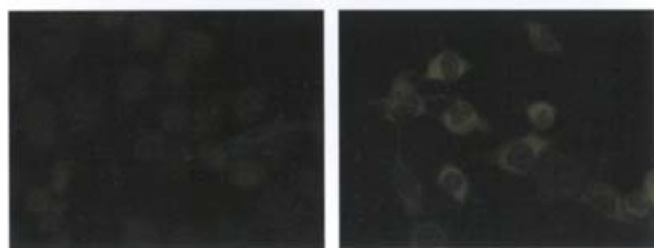

Figure 3. Immunofluorescence staining of XIAP-siRNA transfected H1299/ $\mathrm{m} p 53$ cells with the active caspase- 3 antibody. Cells were incubated at $37^{\circ} \mathrm{C}$ for $24 \mathrm{~h}$ after exposure to $10 \mathrm{~Gy}$ of X-rays. The control cells were not exposed to radiation.

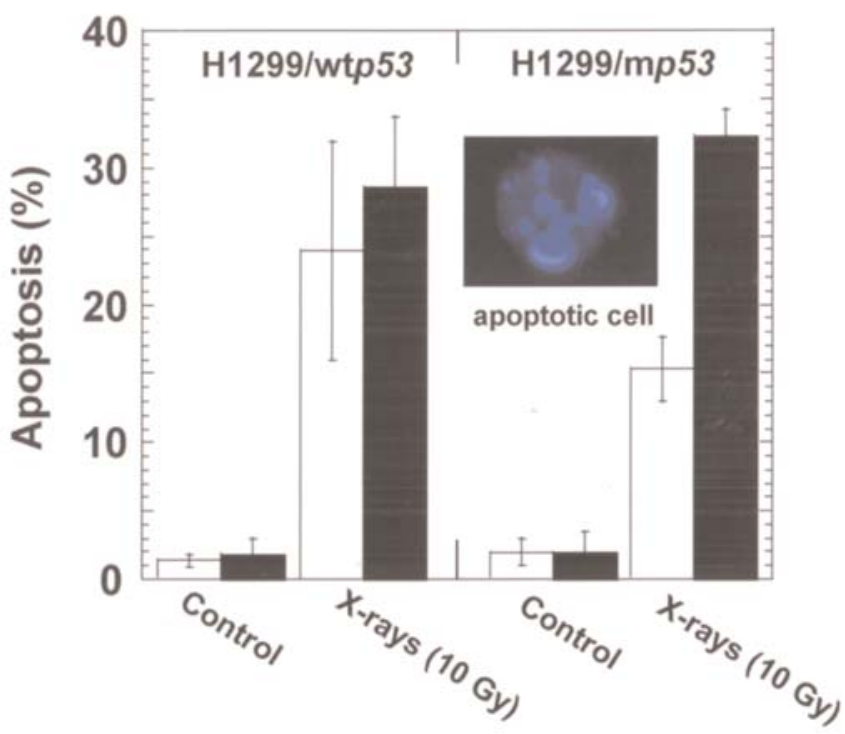

Figure 4. Effects of XIAP-siRNA on X-ray-induced apoptosis. The H1299/ wtp53 and H1299/mp53 cells were irradiated with X-rays (10 Gy) and incubated for $48 \mathrm{~h}$ at $37^{\circ} \mathrm{C}$. After incubation, apoptotic bodies were detected using Hoechst 33342 staining. Open columns, cells transfected with negative control siRNA; closed columns, cells transfected with XIAP-siRNA. The inset photograph shows representative apoptotic bodies. The control cells were not exposed to X-rays.

with increasing time after $\mathrm{X}$-irradiation when compared to non-irradiated cells (Fig. 2).

Detection of active caspase-3 with immunofluorescent staining. Immunofluorescent staining was used to detect active caspase-3 in H1299/mp53 cells after X-irradiation. As shown in Fig. 3, cells positive for the anti-active caspase-3 antibody were observed in the cytoplasm of XIAP-siRNA-transfected and irradiated cells, while such positive cells were almost completely absent in non-irradiated cells. Negative control siRNA-transfected cells did not show any positive cells regardless of $\mathrm{X}$-irradiation status.

Effect of XIAP-siRNA on X-ray-induced apoptosis. Nuclear morphology was analyzed with Hoechst 33342 staining and 
the number of cells showing apoptotic bodies such as those indicated in the insert in Fig. 4 was scored. The incidence of apoptosis $48 \mathrm{~h}$ after treatment with either negative control SiRNA or XIAP-siRNA was $2 \%$ in the H1299/wtp53 and H1299/mp53 cells. X-irradiation after transfection of negative control siRNA induced apoptosis more frequently in the H1299/wtp53 cells ( 24\%) than in the H1299/mp53 cells $(\sim 15 \%)$. In contrast, X-irradiation delivered to cells transfected with XIAP-siRNA enhanced the incidence of apoptosis in the H1299/mp53 cells ( 33\%).

\section{Discussion}

To confirm a previous report that the down-regulation of XIAP expression causes an enhanced radiation sensitivity in lung cancer cells (H1299) (1), the effects of XIAP-siRNA on radiation sensitivity in the $\mathrm{H} 1299$ cells were firstly examined. As shown in Fig. 1a, radiation sensitivity increased in the H1299/wt $p 53$ cells and this effect was even more pronounced in the H1299/mp53 cells transfected with XIAP-siRNA. Western blot analysis (Fig. 1b) showed that XIAP expression was down-regulated in the XIAP-siRNA-transfected cells. These results indicate that the down-regulation of XIAP expression leads to the enhancement of radiation sensitivity in the H1299 cells. Notably, a higher degree of sensitization to radiation was achieved in the H1299/mp53 cells when compared to the H1299/wtp53 cells. Conceivably this may have been due to a higher transfection efficiency in the H1299/mp53 cells than in the H1299/wtp53 cells. However, no difference in transfection efficiency between the H1299/ mp53 and H1299/wtp53 cells was observed. The high degree of sensitization to irradiation in the H1299/mp53 cells appears to be due to the p53 status of the cells.

The enhancement of radiation-induced apoptosis by $X I A P$-siRNA was then examined by looking for the presence of active caspase-3 and apoptotic bodies. Western blot analysis showed that radiation-induced activation of caspase- 3 was increased by the presence of XIAP-siRNA in the H1299/ wtp53 and H1299/mp53 cells (Fig. 2). This result suggests that transfection of XIAP-siRNA increases $p 53$-independent radiation-induced activation of caspase-3. The cleavage of PARP, a marker for caspase- 3 activity, was also enhanced by $X I A P$-siRNA, confirming that the stronger bands observed in the Western blots of caspase-3 are an active form. In agreement with Western blot data, immunofluorescent staining showed a clear increase in positive cells for active caspase-3 when the H1299/mp53 cells were transfected with XIAPsiRNA and irradiated with X-rays (Fig. 3). Apoptotic bodies detected with Hoechst 33342 staining are more frequently observed in the H1299/wtp53 cells than in the H1299/mp53 cells after irradiation alone: H1299/mp53 cells showed a slightly increased rate of apoptosis after irradiation alone. This result is also consistent with Western blot data showing that caspase-3 was activated after irradiation in H1299/wtp53 though not in the H1299/mp53 cells. These results show radiation-induced apoptosis is partially p53-dependent, but that $p 53$-independent apoptosis is also involved in radiationinduced apoptosis to some extent. With the combination of $X I A P$-siRNA and radiation, the enhanced apoptosis is $p 53$ independent and caspase- 3 appears to be involved in this enhanced apoptosis. Some reports have indicated that caspase- 3 is activated independently of p53 through JNK (22), ceramide (23) or Bax (24) during the sensitization of cells to radiation. From these reports, it appears possible that these factors may be involved in the enhancement of radiation-induced caspase- 3 activation by XIAP-siRNA. In contrast to the results reported here, Kappler et al (25) reported that radiosensitization, after a combined treatment of survivin siRNA and irradiation, is correlated with the activation of caspases- 3 and -7 in a wtp53 sarcoma cell line, though not in a mp53 sarcoma cell line. The contradiction in the results between this study and that of Kapppler et al (25) may be due to the different genetic backgrounds of the cell lines used for the two different studies.

Signaling factors inhibiting apoptosis have recently become targets of interest in investigations involving siRNAmediated enhancement of radiation sensitivity $(1,17,25-29)$. This study further supports the potential use of siRNA in targeting an inhibitor of apoptosis as a novel radiation sensitizer for improved radiation cancer therapy, especially in mp53 cells. Radiation cancer therapies based on predictive assays are being examined in patients with a normal p53 function. However, radiation cancer therapeutic strategies against mp53 cancer cells are not well developed. The present study suggests that $X I A P$-siRNA is a possible good candidate for a radiation sensitizer in cancer therapy against $\mathrm{m} p 53$ cells, though it is not known why radiation sensitization by XIAPsiRNA is more effective in the mp53 cells than the wtp53 cells. However, since a high expression of XIAP is clinically associated with a poor survival prognosis in patients (16), $X I A P$-siRNA-mediated enhancement of radiation sensitivity could be more effective in mp53 cells which are associated with a poor prognosis (14).

\section{References}

1. Ohnishi K, Scuric Z, Schiestl RH, Okamoto N, Takahashi A and Ohnishi T: siRNA targeting NBS1 or XIAP increases radiation sensitivity of human cancer cells independent of TP53 status. Radiat Res 166: 454-462, 2006.

2. Ohnishi K, Ota I, Takahashi A and Ohnishi T: Glycerol restores p53-dependent radiosensitivity of human head and neck cancer cells bearing mutant. Br J Cancer 83: 1735-1739, 2000.

3. Ohnishi K, Ota I, Takahashi A, Yane K and Ohnishi T: Transfection of mutant p53 gene depresses X-ray- or CDDPinduced apoptosis in a human squamous cell carcinoma of the head and neck. Apoptosis 7: 367-372, 2002.

4. Ota I, Ohnishi K, Takahashi A, et al: Transfection with mutant p53 gene inhibits heat-induced apoptosis in a head and neck cell line of human squamous cell carcinoma. Int J Radiat Oncol Biol Phys 47: 495-501, 2000.

5. Bristow RG, Jang A, Peacock J, Chung S, Benchimol S and Hill RP: Mutant p53 increases radioresistance in rat embryo fibroblasts simultaneously transfected with HPV16-E7 and/or activated H-ras. Oncogene 9: 1527-1536, 1994.

6. Lotem J and Sachs L: Regulation by bcl-2,c-myc, and p53 of susceptibility to induction of apoptosis by heat shock and cancer chemotherapy compounds in differentiation-competent and defective myeloid leukemic cells. Cell Growth Differ 4: 41-47, 1993.

7. Lotem J and Sachs L: Control of sensitivity to induction of apoptosis in myeloid leukemic cells by differentiation and bcl-2 dependent and independent pathways. Cell Growth Differ 5: 321-327, 1994.

8. Mcllwrath AJ, Vasey PA, Ross GM and Brown R: Cell cycle arrests and radiosensitivity of human tumor cell lines: dependence on wild-type p53 for radiosensitivity. Cancer Res 54: 3718-3722, 1994. 
9. Li R, Sutphin PD, Schwartz D, et al: Mutant p53 protein expression interferes with p53-independent apoptotic pathways. Oncogene 16: 3269-3277, 1998.

10. Ishikawa H, Mitsuhashi N, Sakurai H, Maebayashi K and Niibe H: The effects of p53 status and human papillomavirus infection on the clinical outcome of patients with stage IIIB cervical carcinoma treated with radiation therapy alone. Cancer 91: 80-89, 2001.

11. Harima Y, Harima K, Shikata N, Oka A, Ohnishi T and Tanaka Y: $\mathrm{Bax}$ and $\mathrm{Bcl}-2$ expressions predict response to radiotherapy in human cervical cancer. J Cancer Res Clin Oncol 124: 503-510, 1998.

12. Baldwin AS: Control of oncogenesis and cancer therapy resistance by the transcription factor NF-kappaB. J Clin Invest 107: 241-246, 2001.

13. Perona R and Sanchez-Perez I: Control of oncogenesis and cancer therapy resistance. Br J Cancer 90: 573-577, 2004.

14. Lu B, Mu Y, Cao C, et al: Survivin as a therapeutic target for radiation sensitization in lung cancer. Cancer Res 64: 2840-2845, 2004.

15. LaCasse EC, Baird S, Korneluk RG and MacKenzie AE: A novel anti-apoptosis gene: The inhibitors of apoptosis (IAPs) and their emerging role in cancer. Oncogene 17: 3247-3259, 1998.

16. Tamm I, Richter S, Oltersdorf D, et al: High expression levels of $\mathrm{x}$-linked inhibitor of apoptosis protein and survivin correlate with poor overall survival in childhood de novo acute myeloid leukemia. Clin Cancer Res 10: 3737-3744, 2004.

17. Kim DW, Seo SW, Cho SK, et al: Targeting of cell survival genes using small interfering RNAs (siRNAs) enhances radiosensitivity of Grade II chondrosarcoma cells. J Orthop Res (In press).

18. Cao C, Mu Y, Hallahan DE and Lu B: XIAP and survivin as therapeutic targets for radiation sensitization in preclinical models of lung cancer. Oncogene 23: 7047-7052, 2004.

19. Riedl SJ, Renatus M, Schwarzenbacher R, et al: Structural basis for the inhibition of caspase-3 by XIAP. Cell 104: 791-800, 2001 .
20. Suzuki Y, Nakabayashi Y, Nakata K, Reed JC and Takahashi R $\mathrm{X}$-linked inhibitor of apoptosis protein (XIAP) inhibits caspase-3 and -7 in distinct modes. J Biol Chem 276: 27058-27063, 2001.

21. Takahashi A, Matsumoto H, Yuki K, et al: High-LET radiation enhanced apoptosis but not necrosis regardless of p 53 status. Int $\mathbf{J}$ Radiat Oncol Biol Phys 60: 591-597, 2004.

22. Inanami O, Takahashi $\mathrm{K}$ and Kuwabara $\mathrm{M}$ : Attenuation of caspase-3-dependent apoptosis by Trolox post-treatment of Xirradiated MOLT-4 cells. Int J Radiat Biol 75: 155-163, 1999.

23. Hara S, Nakashima S, Kiyono T, Sawada M, Yoshimura S, Iwama $\mathrm{T}$ and Sakai $\mathrm{N}$ : Ceramide triggers caspase activation during gamma-radiation-induced apoptosis of human glioma cells lacking functional p53. Oncol Rep 12: 119-123, 2004.

24. Lee YJ, Chung DY, Lee SJ, Ja Jhon G and Lee YS: Enhanced radiosensitization of p53 mutant cells by oleamide. Int J Radiat Oncol Biol Phys 64: 1466-1474, 2006.

25. Kappler M, Taubert H, Bartel F, et al: Radiosensitization, after a combined treatment of survivin siRNA and irradiation, is correlated with the activation of caspases 3 and 7 in a wt-p53 sarcoma cell line, but not in a mt-p53 sarcoma cell line. Oncol Rep 13: 167-172, 2005.

26. Guan HT, Xue XH, Dai ZJ, Wang XJ, Li A and Qin ZY: Downregulation of survivin expression by small interfering RNA induces pancreatic cancer cell apoptosis and enhances its radiosensitivity. World J Gastroenterol 12: 2901-2907, 2006.

27. Kami K, Doi R, Koizumi M, et al: Downregulation of survivin by siRNA diminishes radioresistance of pancreatic cancer cells. Surgery 138: 299-305, 2005

28. Rodel F, Hoffmann J, Distel L, et al: Survivin as a radioresistance factor, and prognostic and therapeutic target for radiotherapy in rectal cancer. Cancer Res 65: 4881-4887, 2005.

29. Yano J, Hirabayashi K, Nakagawa S, et al: Antitumor activity of small interfering RNA/cationic liposome complex in mouse models of cancer. Clin Cancer Res 10: 7721-7726, 2004. 